\title{
Unexplained Severe Lactic Acidosis in Young Patient.
}

\author{
Alfred Ibrahimi ${ }^{1}$, Saimir Kuci'1, Ervin Bejko', Stavri Llazo ${ }^{1}$ Maksi \\ Llambro ${ }^{2}$ \\ https://doi.org/10.32391/ajtes.v3i2.55
}

\begin{abstract}
Case report: A 27-year-old previously healthy man was admitted to the ICU complaining nausea, vomiting and disorientation, after he ingested $1500 \mathrm{mg}$ paracetamol, but its relatives explained that they possess in the house only metformin (maybe he ingested metformin instead of paracetamol). First blood gas showed moderate, and hours later severe lactic acidosis $\mathrm{Ph}$ 7,01, Lac $25 \mathrm{~m} \mathrm{~mol} / \mathrm{L}$, BE -26,4. Aggressive rehydration and hemofiltration was started, until full recovery after 24 hours of hospital admission. He was discharged after three days in healthy condition.

Discussion: Lactic acidosis is a common cause of metabolic acidosis at the ICU. Type A is most common and caused by hypoperfusion or hypoxia, whilst type B has other causes including use of the antidiabetic drug metformin. Metformin associated lactic acidosis (MALA) is an important treatment-associated condition, and although rare, it is very serious.

Conclusion: MALA should be strongly suspected in patients presenting with wide anion gap metabolic acidosis and high blood lactate concentration. Bicarbonate hemodialysis or continuous renal replacement therapy should be urgently arranged for patients with MALA.

Keywords: Lactic Acidosis, metformin, hemofiltration.
\end{abstract}

${ }^{*}$ Corresponding Author: Alfred Ibrahimi

$=凶$ E-mail: alfredibrahimi@hotmail.com

${ }^{1}$ Anesthesia Intensive Care Service, German Hospital Tirana, Albania.

${ }^{2}$ Cardiology Service, German Hospital Tirana, Albania. 


\section{Full Text}

\section{Introduction}

Lactate levels greater than $2 \mathrm{mmol} / \mathrm{L}$ represent hyperlactatemia, whereas lactic acidosis is generally defined as a serum lactate concentration above 4 $\mathrm{mmol} / \mathrm{L}$. Lactic acidosis is the most common cause of metabolic acidosis in hospitalized patients. Although the acidosis is usually associated with an elevated anion gap, moderately increased lactate levels can be observed with a normal anion gap (especially if hypoalbuminemia exists and the anion gap is not appropriately corrected). When lactic acidosis exists as an isolated acid-base disturbance, the arterial $\mathrm{pH}$ is reduced. However, other coexisting disorders can raise the $\mathrm{pH}$ into the normal range or even generate an elevated $\mathrm{pH}$. Lactic acidosis occurs when lactic acid production exceeds lactic acid clearance. The increase in lactate production is usually caused by impaired tissue oxygenation, either from decreased oxygen delivery or a defect in mitochondrial oxygen utilization. ${ }^{1}$

\section{Case report}

A young 27 years old man with previously good healthy, presented in hospital complaining, initially nausea and later vomiting, also little bit disorientation and inability to stand still. Physical examination was unremarkable, with normal chest and heart examinations. Heart rate was 100 per min and blood pressure $110 / 70 \mathrm{mmHg}$. Respiratory rate was a little bit higher; 15-18 min. His oral mucosa and tongue was dry and pallid face.
Initially we cannulate cubital venae and started normal saline solution for hydration, supposing that a patient was in first stade of dehydration. One hour later patient started to complain for abdominal pain and in auscultation was observed hyperperistalsis. Respiratory rate increased to 20 without changing in heart rate and blood pressure.

In first blood gas immediately, after he was admitted in hospital, there was a slight metabolic acidosis $(\mathrm{pH} 7,432$. BE 4,8 ) but hours later he profoundly developed moderate to severe lactic acidosis (pH 7.255 lac $13 \mathrm{~m} \mathrm{~mol} / \mathrm{l}, \mathrm{BE}-$ 15,5 ) and glucose level $72 \mathrm{~m} / \mathrm{dl}$. Blood tests on admission showed neutrophil leukocytosis at $12.5 \times 109 / \mathrm{L}$ and slight elevated C-reactive protein.

The patient didn't tell any precise information about what did he had consumed the day before, only a pizza with mushrooms afternoon and $1500 \mathrm{mg}$ acetaminophen for the headache. He didn't complain for any fever, but he was a heavy smoker (50 cigarettes per day).

To exclude splanchnic hypo perfusion we perform a thoracic abdominal TAC which was normal, with abdominal organ well perfused.

After that the patient was sent back to ICU and again we started an aggressive rehydration. ( $3 \mathrm{~L}$ cristaloid solution in 3 hours).

Urinary catheter was inserted with 200 $\mathrm{ml}$ residual urine. For continuous epigastric pain $5 \mathrm{mg}$ iv morphine and 30 $\mathrm{mg}$ papaverine iv was injected.

Despite this treatment, lactic acidosis became more severe $\mathrm{pH} 7.01 \mathrm{BE}-26,4 \mathrm{Lac}$ $26 \mathrm{~m} \mathrm{~mol} / 1$. After insertion of $12 \mathrm{FR}$ two 
lumen Arrow catheter, in left subclavian vein CVVHDF was started, in prismaflex machine (Gambro-Sweden) with M-100 AN-69 membrane. In table 1 there is a progression a blood gas during the period of treatment. After CVVHD was started we saw a good improvement of clinic situation blood gas analysis. Lactate level after 20 hour became normal with no more bicarbonate deficit. We stopped hemofiltration because of the good urinary output without stimulation.
In the next day, patient started to enteral nutrition and he felted well like the day was before the illness.

The patient, during interrogation, denied any drug abuse and insisted on acetaminophen consumption in the house, but his relatives confirmed that they didn't possess any acetaminophen in the house, but only metformin $500 \mathrm{mg}$ of the mother, because she suffered from diabetes type 2. In the third day patient was discharged from hospital in perfect condition.

\begin{tabular}{|c|c|c|c|c|c|c|c|c|c|c|c|c|}
\hline Gas \Time & $\begin{array}{l}10.49 \\
p m\end{array}$ & $\begin{array}{l}2.40 \\
a m\end{array}$ & $\begin{array}{l}3.36 \\
a m\end{array}$ & $\begin{array}{l}7.27 \\
a m\end{array}$ & $\begin{array}{l}8.35 \\
a m\end{array}$ & $\begin{array}{l}9.35 \\
a m\end{array}$ & $\begin{array}{l}10.4 \\
a m\end{array}$ & $\begin{array}{l}10.36 \\
a m\end{array}$ & $\begin{array}{l}12.12 \\
p m\end{array}$ & $\begin{array}{l}1.43 \\
p m\end{array}$ & $\begin{array}{l}5.49 \\
p m\end{array}$ & $\begin{array}{l}8.59 \\
p m\end{array}$ \\
\hline$p h$ & 7.327 & 7.257 & 7.255 & 7.01 & 7.18 & 7.132 & 7.09 & 7.176 & 7.263 & 7.307 & 7.378 & 7.392 \\
\hline Pco2 & 40.5 & 26.9 & 26.2 & 18.7 & 26 & 27.5 & 30.1 & 30.1 & 33 & 30.8 & 32.9 & 36 \\
\hline $\begin{array}{l}\text { Po2(mmHg } \\
\text { ) }\end{array}$ & 33.5 & 102 & 99.1 & 121 & 144 & 129 & 147 & 137 & 136 & 126 & 126 & 128 \\
\hline$c t H B(g / d l)$ & 15.1 & 15.5 & 14.2 & 15.9 & 15 & 14.9 & 15.4 & 15.3 & 14.7 & 13.4 & 13.2 & 11.7 \\
\hline $\operatorname{Hct}(\%)$ & 46.4 & 47.5 & 43.5 & 48.8 & 45.9 & 45.6 & 47.4 & 46.8 & 44.9 & 41 & 40.6 & 35.9 \\
\hline Chco3(m & 21.2 & 14.2 & 13.9 & 8.4 & 9.8 & 11.4 & 11.1 & 12.8 & 15.9 & 16.7 & 19.3 & 21.8 \\
\hline SO2 & 65.8 & 97.8 & 97.3 & 97.2 & 98.4 & 98.5 & 98.6 & 98.8 & 99.3 & 99.3 & 99.4 & 99.6 \\
\hline $\mathrm{K}+(\mathrm{mmol})$ & 3.8 & 5 & 5.1 & 5.6 & 5.4 & 5.7 & 4.9 & 4.8 & 4.9 & 5.4 & 4.8 & 4.4 \\
\hline
\end{tabular}

Table 1: Progression of blood gas

\begin{tabular}{ll}
\hline Amphetamine & $n e g$ \\
\hline Barbiturates & $n e g$ \\
\hline Cannabinoids (Marihuana, Hashish) & $n e g$ \\
\hline Opiates (morphine, heroine, codeine) & $<300 \mathrm{ng} / \mathrm{ml}$ \\
\hline Benzodiazepines & $n e g$ \\
\hline Methaqualone & $n e g$ \\
\hline Propoxyphene & $n e g$ \\
\hline Phencyclidine(PCP) & $n e g$ \\
\hline Methadone (dolophne) & $n e g$ \\
\hline Cocaine Metabolite & $n e g$ \\
\hline Ketone & $30 \mathrm{mg} / \mathrm{dl}$ \\
\hline
\end{tabular}

Table 2:

Urinalysis test 


\section{Discussion}

Lactic acidosis is a common cause of metabolic acidosis with increased anion gap amongst patients at the intensive care unit. Lactic acid is normally produced in excess by about 20 $\mathrm{mmol} / \mathrm{kg} /$ day, which enters the bloodstream. It is then metabolized mostly via the liver and the kidney. Some tissues can use lactate as a substrate and oxidize it to carbon dioxide ( $\mathrm{C} 02)$ and water, but only the liver and kidney have the necessary enzymes to utilize lactate for the process of gluconeogenesis.

The tissues which normally produce excess lactic acid include the skin, red cells, brain tissue, muscle, and the gastrointestinal (GI) tract. During heavy exercise, it is the skeletal muscles which produce the most excess circulating lactate, which normalizes in the absence of impaired hepatic metabolism. In general, elevated lactate can be the result of increased production, decreased clearance, or both ${ }^{2}$. In 1976, Cohen and Woods presented a subdivision of lactic acidosis into type $\mathrm{A}_{\text {and }} \mathrm{B}^{3}$, making type A represent overproduction of lactate due to tissue hypo perfusion like in sepsis or shock, or from tissue hypoxia. Type B lactic acidosis, on the other hand, is characterized by elevated lactate levels from other causes. An example is metformin induced lactic acidosis, which is not seldom seen in the ICU. The antidiabetic agent metformin may trigger lactic acidosis presumably by inhibiting gluconeogenesis and lowering both hepatic uptake and metabolism of lactate to glucose ${ }^{4}$.
What caused severe lactic acidosis acidosis in our patient?

Regarding the information taken from the patient and its relatives we can suppose a few theories.

First, if we take in consideration that the patient took for its headache $1500 \mathrm{mg}$ acetaminophen divided in hours! Paracetamol (acetaminophen) is one of the most commonly taken drugs in overdose in many areas of the world, and the most common cause of acute liver failure in both the UK and USA.

Paracetamol poisoning can result in lactic acidosis in two different scenarios:

Firstly, early in the course of poisoning and before the onset of hepatotoxicity in patients with massive ingestion; a lactic acidosis is usually associated with coma. Experimental evidence from studies in whole animals, perfused liver slices and cell cultures has shown that the toxic metabolite of paracetamol, N-acetyl-pbenzo-quinone imine, inhibits electron transfer in the mitochondrial respiratory chain and thus inhibits aerobic respiration. This occurs only at very high concentrations of paracetamol, and precedes cellular injury by several hours.

The second scenario in which lactic acidosis can occur is later in the course of acetaminophen poisoning as a consequence of established liver failure. In these patients lactate is elevated primarily because of reduced hepatic clearance, but in shocked patients there may also be a contribution of peripheral anaerobic respiration because of tissue hypoperfusion ${ }^{5}$. 
For high anion gap metabolic acidosis, less frequent cause is the temporary accumulation of the organic acid 5oxoproline. 5- oxoproline is primarily metabolized to glutamate by the enzyme 5 -oxoprolinase and is eliminated by the renal system.

5-oxoprolinemia is classically caused by lack of glutathione. Hereditary 5oxoprolinemia is a result of autosomal recessive deficiencies caused by hereditary error in one of the enzymes involved in the gamma-glutamyl cycle, but these rare cases usually present in childhood with hemolytic anemia and neurological defects.

A diagnosis of 5-oxoprolinemia should be considered in any patient with unexplained metabolic acidosis and a history of therapeutic acetaminophen dosing, particularly those patients with coexisting conditions that may decrease the glutathione stores ${ }^{6}$. Our patient didn't took high doses of acetaminophen, didn't have any comorbidities.

Hepatic enzymes, INR, bilirubin were in normal value during the whole time in hospital. So, patient didn't develope any acute hepatic failure and was physically healthy man before this frightened episode for his life.

Secondly, let, s suppose that is a mushroom poisoning (he had eaten a mushroom pizza the night before). The most common consequence of mushroom poisoning is simply gastrointestinal upset. Most "poisonous" mushrooms contain gastrointestinal irritants that cause vomiting and diarrhea. Some of them like "Amanita phalloide" which contain amatoxin a thermoresistant poison.

Indestion of amatoxin can cause acute hepatic failure ${ }^{7}$.

Some other mushroom possess Orellanine which causes kidney failure within 3 weeks after ingestion, monomethylhydrazine causes brain damage, seizures, gastrointestinal upset, and hemolysis etc. Isolated severe lactic acidosis is not seen in mushroom poisoning.

Thirdly, and most importantly, maybe is oversensitivity of organism to low doses of metformin! Metformin is nowadays the first-line drug of choice for the treatment of adults with type 2 diabetes ${ }^{8}$.

This drug is the sixth most frequently prescribed in the USA ( $>50$ million prescriptions in 2009) and is taken by almost $1.5 \%$ of the Italian population 9,10 . Metformin has an absolute oral bioavailability of 50 to $60 \%$, and gastrointestinal absorption is apparently complete within 6 hours. The distribution of metformin follows a twocompartment open model and the distribution volume is around 63-276 liters. Metformin is not metabolized in the body, and $90 \%$ of absorbed metformin is eliminated unchanged in the urine. Plasma protein binding is negligible, so the drug is dialyzable. Metformin is a safe drug when correctly used in properly selected patients.

The literature has reported numerous side effects of metformin, including gastrointestinal disturbances such as nausea, anorexia, vomiting, diarrhea, abdominal discomfort and the sensation of a metallic taste in the mouth. Impaired absorption of vitamin B12 and folic acid 
11, hemolytic anemia ${ }^{12}$, acute hepatitis ${ }^{13}$, and lactic acidosis are less frequent.

Lactic acidosis is a rare but serious side effect, with an estimated incidence of 0.03 per 1000 patient- years.

In particular, no cases of lactic acidosis (a relatively common side effect of other biguanide compounds) were reported in 347 trials with 70,490 patient-years of metformin use 14 .

The term metformin-associated lactic acidosis (MALA) refers to any case of lactic acidosis that develops in a patient treated with metformin, with no further mechanistic insight.

What are the argument that our patient by mistake took metformin instead of Paracetamol?

a) Gastrointestinal symptoms (nausea, vomiting, abdominal pain).

b) Severe lactic acidosis in one patient that one day before was a healthy person (no sepsis and not low cardiac output).

c) Rapid inset of lactic acidosis 6 hours later after ingestion of tablets.

\section{References}

1. Causes of lactic acidosis Authors: Michael Emmett, MD Harold Szerlip, MD, FACP, FCCP, FASN, FNKF Section Editor: Richard $\mathrm{H}$ Sterns, MD Deputy Editor: John P Forman, MD, MSc

2. Lactic Acidosis Christopher D. Foucher

Robert d) Rapid improvement of blood gas after initiation of hemofiltration (metformin is dialyzable drug).

e) Metformin possession in the house $500 \mathrm{mg}$ (like paracetamol) because his mother suffered from diabetes type 2 .

Arguments against MALA:

a) The patient denied metformin ingestion.

b) Low dose of metformin (in literature there are a cases with MALA with high doses of metformin).

c) Measuring metformin level in blood was not possible in hospital.

\section{Conclusion}

In unexplained severe lactic acidosis type B should be strongly suspected metformin overdosing. Bicarbonate, rapid hydration and early hemodialysis or continuous renal replacement therapy should be urgently arranged for patients with MALA. 
lactic acidosis. J Emerg Med 40:271-5.

5. Br J Clin Pharmacol. 2011 Jan;71(1):20-8. doi: 10.1111/j.13652125.2010.03765.x.

6. Understanding lactic acidosis in paracetamol (acetaminophen) poisoning. Shah AD1, Wood DM, Dargan PI.

7. J Med Case Rep. 2014; 8: 409.Published online 2014 Dec 6. doi: 10.1186/1752-1947-8- 409 Acetaminophen-induced anion gap metabolic acidosis secondary to 5-oxoproline: a case report Tarig Mohammed Abkur, Waleed Mohammed, Mohamed Ali, and Liam Casserly

8. Amatoxin Mushroom ToxicityB Z. Horowitz; Michael J. Moss.Author InformationLast

9. Update: November 22, 2018.

10. Nathan DM, Buse JB, Davidson MB, Ferrannini E, Holman RR, Sherwin R, Zinman B. American Diabetes Association; European Association for Study of Diabetes. Medical management of hyperglycemia in type 2 diabetes: consensus algorithm for the initiation and adjustment of therapy: consensus statement of the American Diabetes Association and the European Association for the Study of Diabetes. Diabetes Care. 2009;32:193- 203. doi: $10.2337 / \mathrm{dc} 08-9025$.

11. Top 15 US Pharmaceutical Products by Dispensed Prescriptions.

http://www.imshealth.com/por tal/site/imshealth
12. Gruppo di lavoro OsMed. L'uso dei farmaci in Italia. Rapporto nazionale gennaio- settembre 2010. Rome. 2010.

13. Tomkin G. Malabsorption of vitamin B12 in diabetic patients treated with phenformin: a comparison with metformin. $\mathrm{Br}$ Med J 1973; 3: 673-5.

14. Kashyap AS, Kashyap S. Hemolytic anemia due to metformin. Postgrad Med J 2000; 76: 125-6.

15. Babich MM, Pike I, Shiffman ML. Metformin-induced acute hepatitis. Am J Med 1998; 104: 490-2.

16. Salpeter SR, Greyber E, Pasternak GA, Salpeter EE: Risk of fatal and nonfatal lactic acidosis with metformin use in type 2 diabetes mellitus. Cochrane Database Syst Rev 2010, 4:CD002967. 